\title{
SERIE DE CASOS \\ Distrofia simpática refleja (síndrome doloroso regional complejo tipo-I) asociada a Norplant $®($ L-Norgestrel) \\ Presentación del primer caso y revisión de la literatura \\ Alonso A. Ruiz Perea*, Iván Jaramillo Arias**
}

Recibido: Julio 11/2001 - Revisado: Septiembre 3/2001 - Aceptado: Octubre 2/2001

\begin{abstract}
RESUMEN
El dispositivo subdérmico NORPLANT® (L-Norgestrel) ha sido extensamente utilizado como anticonceptivo en todo el mundo desde hace más de quince años, demostrando ser un método reversible, seguro, efectivo y de alta aceptación por las usuarias. Entre las escasas complicaciones locales se destacan dolor, hematomas, infecciones, expulsión del implante y diversas lesiones neurológicas. Hasta la fecha no se ha informado en la literatura (MEDLINE hasta Diciembre de 2000) ningún caso de Distrofia Simpática Refleja (Síndrome Doloroso Regional Complejo Tipo-I) asociado a la utilización de este moderno método.

Se presenta el caso de una usuaria de este dispositivo, que presentó un cuadro característico de Distrofia Simpática Refleja en el brazo izquierdo después de colocársele un NORPLANT® (L-Norgestrel), que evolucionó desde el momento de su inserción hasta su extracción tres años después, con remisión total del Síndrome doloroso.
\end{abstract}

PALABRAS CLAVE: NORPLANT®, distrofia simpática refleja.

\section{SUMMARY}

NORPLANT® (L-Norgestrel) has been widely used worldwide during the last fifteen years, proving to be a reversible, safe, effective, highly-accepted contraceptive implant method for its users. Among the few local, minor complications derived from its use, it is important to mention pain, hematoma, infections, implant device expelling and some neurological injuries. To date (Decembre 2000), according to MEDLINE search engine no case of Reflex Sympathetic Syndrome (Complex Regional Pain Syndrome Type-I) related to the use of this modern contraceptive method has ever been reported in medical literature.

The authors introduce the case of an user who presented in her left arm a characteristic clinical picture of Reflex Sympathetic Dystrophy, wich evolved from the moment the implant was inserted until its extraction three years later, with complete remission of the Pain Syndrome.

KEY WORDS: NORPLANT®, Reflex sympathetic dystrophy.

* Médico Especialista en Medicina Interna de la Universidad del Cauca. Miembro de la Asociación Colombiana de Medicina Interna. Profesor Asistente Departamento Medicina Interna. Facultad Ciencias de la Salud - Universidad del Cauca. Popayán, Cauca, Colombia.
** Médico Especialista en Ginecología y Obstetricia de la Universidad del Cauca. Miembro de la Sociedad Colombiana de Obstetricia y Ginecología. Popayán, Cauca, Colombia. 


\section{CASO CLÍNICO}

Paciente de 27 años, casada, G1P1, estudiante universitaria, sin antecedentes patológicos de importancia, remitida por Ginecología para evaluación de dolor y edema en el brazo izquierdo. Se le había insertado, sin complicaciones inmediatas un dispositivo de tipo NORPLANT® en el antebrazo izquierdo tres años atrás, por parte de otro ginecólogo. Refería desde entonces y en forma episódica "una molestia" no descrita como franco dolor, de tipo parestesias que se le exacerbaba con los movimientos del brazo, sin cambios en la piel. Su ginecólogo la había tranquilizado afirmándole que dicha sintomatología era normal. El dolor fue en aumento a lo largo de los meses convirtiéndose en dolor tipo quemante y desde 45 días antes de la consulta notaba sensación de hinchazón en antebrazo, brazo y dorso de la mano izquierda, con dolor generalizado tipo quemante en la extremidad, pero más localizado en el pliegue antecubital; en los últimos quince días el dolor se había hecho insoportable, no calmaba con analgésicos comunes y la despertaba en la noche. Sentía permanentemente la extremidad fría, notaba que adquiría una coloración violácea en parches (cianosis) y percibía parestesias en el aspecto cubital del brazo y de la palma de la mano izquierda, relacionadas con el movimiento y con el frío (alodinia mecánica y térmica).

En la Revisión por Sistemas no se encontraron antecedentes de importancia. Su único embarazo había sido bien tolerado, con parto eutócico y a término. Nunca antes había planificado; no había exposición a ningún tóxico, no ingería regularmente ningún medicamento y hacía deporte de manera regular. Sus ciclos menstruales durante el periodo de uso de NORPLANT® (L-Norgestrel) habían sido normales.

Refería como antecedentes familiares ser hija única, padres sanos y abuelo materno diabético.

EXAMEN FÍSICO: Paciente joven, delgada, afebril, álgica, aprehensiva respecto a su estado. T.A. $=126 / 68$ $\operatorname{mmHg}$, F.C. $=74 \times \min$, F.R. $=12 \times \min , \mathrm{T} .=36.7^{\circ} \mathrm{c}, \mathrm{P} .=$ $59 \mathrm{Kg}$., I.M.C. $=22.6 \mathrm{Kg} / \mathrm{m} 2$.

Cabeza: normal. ORL: normal. Cuello normal y fosas supraclaviculares libres. Cardiopulmonar y abdomen: normal. Ganglionar: negativo. Extremidades: en antebrazo y brazo izquierdos se encuentra piel caliente, edematosa en parches, engrosada y seca desde el hombro hasta los dedos de la mano, con "cutis marmorata" y con áreas en las que la piel es más fría; disminución de los arcos de extensión, flexión y rotación del hombro y dolor a la movilización articular sin detectarse derrame ni sinovitis. Se encuentra pequeña cicatriz puntiforme en el sitio de inserción del NORPLANT® a $4 \mathrm{~cm}$ por encina del pliegue antecubital izquierdo con edema local, pero sin otros signos inflamatorios ni áreas renitentes. Diámetro del brazo a $10 \mathrm{~cm}$ por encima del epicóndilo: $20 \mathrm{~cm}$ (izquierdo) y $18 \mathrm{~cm}$ (derecho); diámetro del antebrazo a 10 $\mathrm{cm}$ por debajo del epicóndilo: $15 \mathrm{~cm}$ (izquierdo), $14 \mathrm{~cm}$ (derecho). Dolor intenso a la movilización del codo, sin sinovitis ni derrame local. Edema difuso de los dedos de la mano izquierda dando el aspecto "en salchichas" de los mismos, con retardo en el llenado capilar, con pulsos radiales y cubitales simétricos. El examen neurológico fue normal, excepto por hipoestesia en parches a lo largo del territorio del dermatoma C4-C5 e hiperestesia cutánea en antebrazo y brazo izquierdos.

Con este cuadro clínico se planteó el Diagnóstico de DISTROFIA SIMPÁTICA REFLEJA (SÍNDROME DOLOROSO REGIONAL COMPLEJO TIPO-I) asociada a NORPLANT®. El hemograma con eritrosedimentación fue normal, al igual que Glicemia, Creatinina y pruebas hepáticas. Se le efectuó Electromiografía y Neuroconducción Comparativa, las cuales fueron totalmente normales, descartándose cualquier lesión neurológica. Se le propuso al ginecólogo tratante su extracción, procedimiento que se efectuó ambulatoriamente en el consultorio 4 días después, Se extrajeron fácilmente 5 de las barras, siendo más complicada y dispendiosa la extracción de la $6^{\mathrm{a}}$, la cual había migrado ligeramente en dirección axilar. Se le inició manejo con Terapia Física, Amitriptilina a $37.5 \mathrm{mg} /$ día y Deflazacort a 60 mg/día (1 mg x Kg x día).

Al día siguiente la paciente presentaba menos dolor, sentía menos hinchado el brazo y las parestesias habían disminuido. Aunque las características de la piel continuaban iguales, el llenado capilar era normal, pero presentaba marcada limitación al movimiento del hombro por una capsulitis adhesiva del mismo, que respondió bien a la Terapia Física. Recibió Deflazacort por 21 días en dosis progresivamente menores y Amitriptilina a la dosis descrita, desapareciendo su sintomatología en el lapso de un mes y medio.

En consultas sucesivas de control hasta 2 años después, el fenómeno doloroso había desaparecido totalmente y continuaba su anticoncepción con anovulatorios orales sin complicaciones. 


\section{DISCUSIÓN}

Se presenta el primer caso de DISTROFIA SIMPÁTICA REFLEJA (SÍNDROME DOLOROSO REGIONAL COMPLEJO TIPO-I), asociada a la implantación de NORPLANT® (L-Norgestrel). La búsqueda de la información se efectuó en MEDLINE hasta Diciembre de 2000, sin registrarse hasta entonces ninguna asociación entre éste método anticonceptivo y la DISTROFIA SIMPÁTICA REFLEJA (SÍNDROME DOLOROSO REGIONAL COMPLEJO TIPO-I).

\section{NORPLANT (L-NORGESTREL)}

NORPLANT® (L-Norgestrel) es un sistema de anticoncepción desarrollado por el Population Council y aprobado por la OMS desde 1984 como una alternativa segura en las opciones anticonceptivas disponibles en la comunidad1. Se ha utilizado desde entonces en más de 700.000 usuarias en casi todo el mundo. Es un método efectivo, reversible, de acción prolongada (hasta cinco años), que brinda protección anticonceptiva inmediata (desde las primeras 24 horas) y continua por medio de pequeñas cápsulas que contienen solamente progestágeno (Levonorgestrel) en bajas dosis insertadas bajo la piel del antebrazo de la mujer.

En Colombia se inició un estudio clínico de NORPLANT® (L-Norgestrel) en 1982 en el Instituto Materno Infantil de Bogotá y en el Hospital Federico Lleras de Ibagué con casi 390 usuarias, con una tasa de embarazos de $0 \%$ y una tasa de continuación de anticoncepción de $76.5 \%$ a los dos años de su uso ${ }^{2}$. Estudios iniciales en República Dominicana ${ }^{3}$ mostraron una tasa de embarazo, a los dos años, de $0 \%$ y una tasa neta de continuación a los dos años de $88.1 \%$. En Chile ${ }^{4}$ la tasa acumulada de embarazos fue de $3.2 \times 100$ años/mujer a los cinco años de su uso y en Indonesia la tasa acumulada de embarazos en los cinco años fue de $1.8 \mathrm{x}$ cada 100 mujeres 5 .

NORPLANT® (L-Norgestrel) brinda protección anticonceptiva hasta por cinco años, al cabo de los cuales deberá retirarse. Seis delgadas cápsulas fabricadas en silicona que contienen Levonorgestrel se insertan subdérmicamente en el brazo o antebrazo de la mujer. Cada cápsula mide $3.4 \mathrm{~cm}$ de longitud, un diámetro de $0.24 \mathrm{~cm}$ y contiene $36 \mathrm{mg}$ del progestágeno. Cada día se liberan aproximadamente $30 \mathrm{mcg}$ (microgramos) de Levonorgestrel (L-Norgestrel), lo que equivale a la cantidad de L-Norgestrel contenido en la dosis diaria de la píldora de solo progestágeno y es menor que la dosis diaria suministrada por los anticonceptivos orales combinados.

El método se indica prácticamente para cualquier mujer que desee planificar; se contraindica en caso de embarazo o sospecha de estarlo, así como en mujeres con hepatopatías, trastornos trombogénicos, enfermedad oclusiva cerebrovascular o coronaria y en aquellas con sangrado anormal no diagnosticado. Los barbitúricos, fenitoína, isoniacida, carbamacepina y rifampicina pueden disminuir su efectividad anticonceptiva y los efectos colaterales más frecuentes son el cambio del patrón de sangrado menstrual al inicio del tratamiento, cefalea, leve aumento del peso, secreción por el pezón, acné, cambios en el estado anímico y raras veces dolor en el sitio del implante.

La inserción es un procedimiento de consultorio con anestesia local, practicado por un médico, habitualmente un Ginecobstetra debidamente adiestrado y no tarda más de 5-15 minutos. Se efectúa una pequeña incisión de $2 \mathrm{~mm}$, se pasa un trocar para insertar las cápsulas que se colocan debajo de la piel en la cara interna del tercio inferior del brazo o superior del antebrazo y no requiere de sutura posterior. Los efectos locales no van más allá de 0.4 a $4.7 \%$ y puede producirse en los primeros días leve dolor, pequeño hematoma; raras veces infecciones, expulsión del implante y lesiones neurológicas como Neuropatía cubital traumática ${ }^{6,31}$. El momento más apropiado para la inserción es durante la menstruación y a más tardar en el séptimo día de inicio del ciclo menstrual para tener la certeza de la ausencia de embarazo.

\section{La extracción de NORPLANT® (L-Norgestrel)} puede hacerse en cualquier momento del ciclo menstrual, tarda un poco más que la inserción (15-20 minutos) y puede ser un poco más difícil; debe ser extraído por un médico, preferiblemente el Ginecobstetra, bien entrenado y familiarizado con el procedimiento. Una vez palpadas las cápsulas se procede a colocar anestesia local y se extraen a través de una incisión de unos $4 \mathrm{~mm}$ de longitud. El dispositivo se debe extraer a los cinco años de insertado o antes por razones personales o médicas: cefalea "de novo" o empeoramiento de cefaleas previas, alteraciones agudas de la visión o trastornos tromboembólicos, aumento de peso, hemorragias uterinas anormales, mastalgia y ocasionalmente dolor local. El retiro de NORPLANT $®$ (L-Norgestrel) no está exento de riesgos, reportándose Neuropatía cubital ${ }^{7}$ y lesiones neurovasculares diversas, habitualmente no graves. 
Múltiples estudios prospectivos en todo el mundo demuestran que NORPLANT® (L-Norgestrel) es un método muy efectivo como anticonceptivo, con tasas acumulativas de embarazo entre $0 \%$ a los 4 años $^{8}$ y $1.9 \%$ a los 7 años ${ }^{9}$, siendo mayor la frecuencia de embarazo en las mujeres con sobrepeso ${ }^{10}$. Es igualmente seguro, ya que estudios prospectivos no han demostrado alteraciones en metabolismo de la glucosa ${ }^{11}$, en el sistema de la coagulación ni en las pruebas de función hepática ${ }^{12}$, así como tampoco en la mineralización ósea medida por DEXA $^{13}$ ni en el perfil lipídico ${ }^{14}$. La función del eje pituitaria-suprarrenales y la capacidad de reserva adrenal puede verse alterada, con mínima reducción de la reserva adrenal por lo que deberá tenerse cautela durante estrés agudo $\mathrm{o}$ en cirugías prolongadas, según demostraron Toppozada et $\mathrm{al}^{15}$ cuando midieron los niveles de ACTH, Cortisol, 17-OH esteroides y 17-Cetoesteroides los cuales permanecieron prácticamente inalterados, con solo leve reducción de la reserva adrenal.

Un estudio pequeño comenzó demostrando que NORPLANT® (L-Norgestrel) no contribuye al riesgo cardiovascular en las usuarias del sistema ${ }^{16}$; estos hallazgos fueron corroborados más tarde en un extenso estudio multicéntrico en cinco centros clínicos de Bangkok, Yakarta, Ciudad de México, Singapur y Estocolmo $^{17}$, concluyéndose que no se aumenta el riesgo de enfermedad aterosclerótica con el uso prolongado en las usuarias.

Las tasas de aceptación del método varían de país en país y de cultura en cultura: en general es menos aceptado por la población urbana de Nueva York $^{8-9}$, que en la República China ${ }^{10}$, Pakistán ${ }^{18}$, Indonesia o Bangladesh ${ }^{19}$. Se ha establecido que el $50.38 \%$ de las usuarias lo abandonan en los primeros tres años en México ${ }^{20}$ mientras que el $76.5 \%$ en Colombia lo sigue utilizando a los dos años de su inserción ${ }^{2}$.

\section{DISTROFIA SIMPÁTICA REFLEJA (SÍNDROME DOLOROSO REGIONAL COMPLEJO TIPO-I):}

El término Síndrome de Distrofia Simpática Refleja, introducido por Evans en la literatura médica en 1946 ha recibido varias denominaciones, todas ellas imprecisas, denotando algunas el origen del trastorno, otras los mecanismos del dolor y otras la evolución del mismo. Así, se le ha llamado: SÍNDROME DE CAUSALGIA (MAYOR Y MENOR), SÍNDROME HOMBRO-MANO,
ATROFIA DE SUDECK, ALGODISTROFIA, ALGONEURODISTROFIA, DISTROFIA REFLEJA NEUROVASCULAR, SÍNDROME DE STEINBROCKER, OSTEOPOROSIS POSTRAUMÁTICA, DISTROFIA POSTRAUMÁTICA, VASOESPASMO POSTRAUMÁTICO, EDEMA CRÓNICO POSTRAUMÁTICO, SIMPATALGIA, etc. A partir de 1994 la Asociación Internacional para el Estudio del Dolor (International Association for Study of Pain _ IASP, por sus siglas en inglés) reunió todas estas definiciones bajo el término de SÍNDROME DOLOROSO REGIONAL COMPLEJO para denotar el dolor regional y los cambios autonómicos y sensitivos que exceden en intensidad, magnitud o ambos a un fenómeno traumático ${ }^{21-22}$.

Así, el Síndrome Doloroso Regional Complejo quedó clasificado en Tipo-I (Distrofia Simpática Refleja) y en Tipo-II (Causalgia). En este último, el dolor es producido esencialmente por una definida y demostrable lesión del nervio periférico y sus ramas.

El Síndrome Doloroso Regional Complejo Tipo-I o Distrofia Simpática Refleja es más frecuente en personas jóvenes y suele ser causado por traumas mayores o menores habitualmente en las extremidades, fracturas, cirugías ortopédicas o vasculares, lesiones médicas como Osteoartritis, discopatías intervertebrales, Lupus Eritematoso Sistémico con Anticoagulante Lúpico ${ }^{26}$, Infarto Miocárdico o eventos cerebrovasculares; pero a diferencia de la Causalgia, la lesión del nervio periférico $\mathrm{NO}$ es demostrable en la Electromiografía (EMG) ni en los estudios de Neuroconducción. En este caso es característico que la intensidad de los síntomas sea desproporcionada a la severidad del trauma ${ }^{22}$. Aunque la irradiación del dolor suele ser distal al sitio del evento inicial, en $70 \%$ de los casos el dolor se irradia a áreas distantes, no contiguas, sin un patrón neuropático y sin seguir la zona de inervación de un dermatoma; y puede ser tan caprichosa como la diseminación "en espejo" en la extremidad contralateral sana ${ }^{23}$. Algunos casos pueden aparecer espontáneamente, sin un evento precipitante definido.

El dolor habitualmente se describe como tipo quemante o urente, asociado a rigidez muscular o articular, sensación de edema, trastornos en la sudoración local y alteraciones de la sensibilidad como alodinia térmica, hiperalgesia y disestesias y es frecuente encontrar eritema localizado en esta primera fase. Con el tiempo el dolor se intensifica (segunda fase), la alodinia térmica es mayor, aparece edema de la extremidad, con cambios en la 
coloración de la misma (palidez o cianosis) y severa restricción de los arcos de movimiento articular correspondientes. Posteriormente (tercera fase) aparece atrofia muscular por desuso, atrofia de la dermis y epidermis y contracturas musculares $\mathrm{Si}$ el manejo no se inicia precozmente el cuadro clínico evoluciona casi inevitablemente a la cronicidad.

Es fácil ver con ésta evolución natural de la enfermedad cómo se le ha denominado anteriormente desde cuadros de edema doloroso hasta cuadros de atrofia y por consiguiente las antiguas denominaciones solamente reflejaban el momento de la evolución en que se observaban y se diagnosticaban las lesiones.

El término más empleado ha seguido siendo, a pesar de todo, el de Distrofia Simpática Refleja. Sin embargo, ¿cuál es el papel del Simpático en éste fenómeno doloroso?. La principal función del Sistema Nervioso Simpático es la de preparar al individuo para enfrentar los estímulos adversos o amenazantes de la vida en el medio ambiente $^{21}$. La experiencia clínica ha demostrado, sin embargo, que el Simpático puede ser el responsable de mantener y perpetuar ciertos síndromes dolorosos crónicos, particularmente aquellos en que el dolor mejora o desaparece al efectuar un bloqueo simpático ${ }^{21-22,24}$.

Las siguientes observaciones indican que las acciones eferentes del Simpático están involucradas en la generación y mantenimiento de ciertos estados dolorosos:

- La función simpática en el área afectada es anormal: edema, sudoración y distrofia en la piel (trastorno sudomotor) y alteraciones del flujo sanguíneo con cambios en la temperatura (trastorno vasomotor).

- Pronto alivio del dolor tras una terapia simpaticolítica, en aquellos casos con dolor mediado por el simpático (no son todos los casos).

- Activación del sistema nervioso simpático por ruidos intensos, aplicación Iontoforética o aplicación intradérmica de agentes adrenérgicos, que exacerban el dolor.

Sin embargo se ha demostrado que el dolor en la Distrofia Simpática Refleja no siempre es generado, mantenido ni perpetuado por el Sistema Nervioso Simpático ${ }^{24-25,} 27-28$, razón por la cual se cambió su denominación por Síndrome Doloroso Regional Complejo, aunque se puede considerar que existen normalmente interacciones simpáti- co-sensitivas en los tejidos sanos en el humano, demostrándose que la actividad simpática y las catecolaminas pueden activar primariamente los nociceptores aferentes ${ }^{27}$. De aquí que se haya reportado tanto fracaso terapéutico con solo Simpaticolíticos en los casos de Síndrome Doloroso Regional Complejo Tipo-I o Distrofia Simpática Refleja.

Se ha tratado de investigar qué tanto factor inflamatorio podría existir en el Síndrome Doloroso Regional Complejo Tipo-I; sin embargo en un estudio reciente en Holanda, la Citometría de flujo en 13 pacientes no mostró diferencias con la de los sujetos controles, por lo tanto no se encontró asociación entre los índices inmunológicos y la Distrofia Simpática Refleja ${ }^{29}$.

Por último, tratando de encontrar un posible factor genético, Kemler $\mathrm{M}$ y colaboradores en Holanda ${ }^{30}$ encontraron que en 52 pacientes cuidadosamente seleccionados con Distrofia Simpática Refleja la frecuencia de HLA-DQ1 fue significativamente superior a los controles, pero este factor genético requiere de mayores estudios.

El caso presentado se ajusta a los criterios de la Asociación Internacional para el Estudio del Dolor (IASP, en inglés) para establecer el Diagnóstico de SÍNDROME DOLOROSO REGIONAL COMPLEJO TIPO I (antes llamado DISTROFIA SIMPÁTICA REFLEJA), toda vez que se trata de un dolor crónico con características neuropáticas desproporcionado al mínimo tramo por la inserción del dispositivo de L-Norgestrel y con estudios de Neuroconducción y Electromiografía normales, excluyéndose así un Síndrome Doloroso Regional Complejo tipo II antes llamado Causalgia, aún sin tener uno de los elementos contundentes como es la respuesta favorable al bloqueo Simpático. Desconocemos si el Simpático inició y/o perpetuó el dolor y qué tanto papel desempeñaron, el fenómeno inflamatorio crónico y el desuso.

\section{CONCLUSIÓN}

NORPLANT® ha demostrado ser un método anticonceptivo reversible, seguro, efectivo y aceptable. Sus efectos sistémicos son escasos y sus complicaciones locales lo son aún más. Aunque se ha reportado Neuropatía periférica y otras lesiones neurológicas menores directamente relacionadas con la implantación, el uso y el retiro del dispositivo, no se había reportado antes en la literatura un caso bien documentado de Distrofia 
Simpática Refleja (Síndrome Doloroso Regional Complejo Tipo-I) asociado a este dispositivo. En consecuencia, se llama la atención a la presenta- ción de ésta complicación que debe tenerse en cuenta para enfocar adecuadamente el diagnóstico e iniciar un manejo rápido y oportuno.

\section{BIBLIOGRAFÍA}

1. International Medical Advisory Pannel. "Statement on NORPLANT® Subdermal Contraceptive Implant System". IPPF Medical Bulletin 1985; 19(6): 1-3.

2. López G, et al. "Two-Years Prospective Study in Colombia of NORPLANT® Implants". Obstet. Gynecol 1986; 68(2): 204-208.

3. Álvarez-Sánchez, et al. "The Clinical Performance of NORPLANT® Implants over Time: A Comparison of Two Cohorts". Studies in Fam Plann 1988; 19(2): 118-121.

4. Sivin I "International Experience with NORPLANT® and NORPLANT®-2 Contraceptives". Studies in Fam Plann, 1998; 19(2): 81-94.

5. Affandi B, et al. "Five Years Experience with NORPLANT®". Contraception 1987; 36(4): 417-428.

6. Marin R; McMillan D. "Ulnar neuropathy associated with subdermal contraceptive implant". South Med Journal. 1998 Sept; 91(9): $875 \_878$.

7. Smith JM; Conwitt RA; Blumenthal PD: "Ulnar nerve injury associated with removal of Norplant implants". Contraception $1998 \mathrm{Feb}$; 57(2): 99-101.

8. Sivin I, et al. "The performance of Levonorgestrel rod and Norplant contraceptive Implants: a 5-year randomized study". Hum Reproduct 1998 Dec; 13 (12): 3371-3378.

9. Sivin I, et al. "Prolonged effectiveness of Norplant capsule implants: a 7-year study". Contraception 2000 Mar; 61(3): 187194.

10. Du MK; Chow LP; Zhen HM; Chen CH. "A 10-year follow-up study of contraceptive Norplant Implants". Int J Gynecol Obstet 2000 Mar; 68(3): 249-256.

11. Sagay AS, et al. "Glucose metabolism among Norplant users in Northern Nigeria". Contraception 2000 Jul; 62(11): 19-22.

12. Egberg N, et al. "Effects on the Hemostatic system and liver function in relation to Implanon and Norplant. A prospective randomized clinical trial". Contraception 1998 Aug; 58(2): 93-98.

13. Di X; LIY; Zhang C; Jiang J, Gu S. "Effects of Levonorgestrelreleasing subdermal contraceptive implants on bone density and bone metabolism" Contraception 1999 Sept; 60(3): 161-166.

14. Singh K; Ratnam SS. "A Study on the effects of Norplant implantable contraceptive on lipid, lipoprotein and apolipoprotein metabolism in Singaporean women". Contraception 1997 Aug; 56(2): 77-83.

15. Toppozada MK; Ramadan M, et al. "Effects of Norplant Implants on the pituitary-adrenal axis function and reserve capacity". Contraception 1997 Jan; 55(1): 7-10.

16. Petiti DB; et al. "Norplant Implants and cardiovascular disease". Contraception 1998 May; 57(5): 361-362.

17. U.N. / W.H.O. "Study of the effects of implantable contraceptive Norplant on lipid and lipoprotein metabolism. UN Development Programme/ UN Population Fund/ W.H.O. / World Bank,
Special Programme of Research, Development and Research Trainning in Human Reproduction, Task Force on Long-Acting Systemic agents for fertility Regulation". Contraception 1999 Jan; 59(1): 33-45.

18. Rehan N; Inayatullah A; Chaudhary G. "Norplant: Reasons for discontinuation and side-effects". Europ J Contracept Reprod Health Care 2000 Jun; 5(2): 113-118.

19. Hardee K; Balogh S; Villinski MT. "Three countries experience with Norplant introduction". Health Policy Plan 1997 Sept; 12(3): 199-213.

20. Del Carmen Cravioto M, et al. "A Multicenter comparative study on the efficacy, safety and acceptability of the contraceptive subdermal implants Norplant and Norplant-II". Contraception 1997 Jun; 55(6): 77-83.

21. Wesselman U; Raja S. "Reflex Sympathetic Dystrophy and Causalgia". Anesthesiol Clin of N A. 1997 Jun; 15(2): 407-427.

22. Wasner G; Backonja MM; Baron R. "Traumatic Neuralgias Complex Regional Pain Syndromes (Reflex Sympathetic Dystrophy and Causalgia): Clinical Characteristics, Pathophysiologic Mechanisms and Therapy". Neurol Clin of N A. 1998 Nov; 16(4): 851-868.

23. Maleki J; LeBel AA; Bennett GJ; Schwartzman RJ. "Patterns of spread in Complex Regional Pain Syndrome Type-I (Reflex Sympathetic Dystrophy)". Pain 2000 Dec 1; 88(3): 259-266.

24. Manning DC: "Reflex Sympathetic Dystrophy, Sympathetically mantained pain, and Complex Regional Pain Syndrome: Diagnosis of Inclusion, Exclusion or Confusion?". Journal of Hand Ther 2000 Oct; 13(4): 260-268.

25. Stanton-Hicks M. "Complex Regional Pain Syndrome (Type-I, RSD; Type-II, Causalgia): Controversies". Clinical Journal of Pain 2000 Jun; 16(2 Suppl): S33-S40.

26. Tsutsumi A, et al. "Reflex Sympathetic Dystrophy in a patient with the antiphospholipid Syndrome". Lupus 1999; 8(6): 471-473.

27. Baron R; Levine JD; Fields HL: "Causalgia and Reflex Sympathetic Dystrophy: Does the Sympathetic nervous system contribute to the generation of pain?" Muscle Nerve 1999 Jun; 22(6): 678-695.

28. Wilson PR. "Post-Traumatic upper extremity Reflex Sympathetic Syndrome. Clinical Course, Staging and Classification of Clinical Forms". Hand Clin 1997 Aug; 13(3): 367-372.

29. Ribbers GM; Oosterhuis; van Limbeek J; de Metz M. "Reflex Sympathetic Dystrophy: Is the Immune System Involved?". Arch Phys Med Rehabil 1998 Dec; 79(12): 1549-1552.

30. Kemler M, et al. "HLA-DRQ1 associated with Reflex Sympathetic Syndrome". Neurology 1999 Oct 12; 53(6): 13501351.

31. Hueston WJ; Locke KTI. "Norplant neuropathy: peripheral neurologic symptoms associated with subdermal contraceptive implants". Journal Fam Pract 1995 Feb; 40(2): 184-186. 